\title{
Efficacy and Safety of Psychostimulants for Alzheimer's Disease: A Systematic Review and Meta-Analysis
}

\author{
Authors \\ Taro Kishi, Kenji Sakuma, Nakao Iwata
}

\begin{abstract}
Affiliations
Department of Psychiatry, Fujita Health University School of Medicine, Toyoake, Aichi, Japan

Keywords

Alzheimer's disease, psychostimulant, systematic review and meta-analysis, apathy, cognition impairment
\end{abstract}

$\begin{array}{ll}\text { received } & 10.10 .2019 \\ \text { revised } & 22.11 .2019 \\ \text { accepted } & 03.12 .2019\end{array}$

Bibliography

DOI https://doi.org/10.1055/a-1076-8228

Published online: 30.1 .2020

Pharmacopsychiatry 2020; 53: 109-114

(c) Georg Thieme Verlag KG Stuttgart · New York

ISSN 0176-3679

Correspondence

Dr. Taro Kishi

Department of Psychiatry

Fujita Health University School of Medicine

1-98 Dengakugakubo, Kutsukake-cho

470-1192 Toyoake, Aichi, Japan

tarok@fujita-hu.ac.jp $\circledast$ Supporting Information for this article is available online at https://doi.org/10.1055/a-1076-8228.

\section{ABSTRACT}

Introduction Several reports of the effectiveness of the use of psychostimulants for the treatment of Alzheimer's disease (AD) are available.

Methods A systematic review and meta-analysis was conducted including double-blind, randomized, placebo-controlled trials. Outcomes were the improvement of apathy scales score (primary), mini-mental state examination (MMSE) score, activities of daily living scale score, Zarit burden interview score, all-cause discontinuation, discontinuation due to adverse events, and incidence of at least 1 adverse event.

Results Three methylphenidate studies and 1 modafinil study were identified ( $n=156)$. Results from combined psychostimulants were superior to placebo in the improvement of apathy scales score (standardized mean differences [SMD] $=-0.63$ $(-1.22,-0.04), p=0.04$, all studies) and the MMSE score $(\mathrm{SMD}=-0.58(-1.14,-0.02), \mathrm{p}=0.04,3$ methylphenidate studies). The modafinil study was excluded from the meta-analysis for the improvement of apathy scales score; therefore, the effect size increased $(S M D=-0.82(-1.43,-0.20), p=0.009)$. However, no significant differences were observed in terms of other outcomes, including safety outcomes between the treatment groups.

Discussion Methylphenidate would be effective in treating apathy and cognitive impairment in $\mathrm{AD}$ patients.

\section{Introduction}

Alzheimer's disease (AD) is a common neurodegenerative disorder. Recently, Chicago health and aging population study showed that the 2010 census standardized prevalence of dementia caused by AD was $14.5 \%$ (95\% confidence interval $[\mathrm{Cl}]=13.7-15.3)$, and the annual incidence rate was $2.3 \%(1.7-2.9)$ [1]. The symptoms of AD are classified into cognitive impairment and behavioral disturbances [2]. Behavioral disturbances include various psychiatric symptoms, such as depression, apathy, psychosis, anxiety, agitation, and sleep disturbances [2]. A recent systematic review and meta-analysis showed that memantine treatment is beneficial for psychosis, agitation, and sleep disturbances in patients with $\mathrm{AD}$ [3]. However, apathy is the most common neurobehavioral symptom associated with $A D$, but currently approved anti-dementia drugs do not improve this symptom [4].

The pathophysiology of apathy includes impairment of dopaminergic neurotransmission in the brain areas, such as the ventral tegmental area $[5,6]$. Prolonged exposure to amyloid oligomers decreases the release of glutamate and gamma-aminobutylic acid, reducing the possibility of dopamine release in the prefrontal cortex and hippocampus [5]. The progressive decrease of glutamate release from the prefrontal cortex reduces the stimulus for dopamine release in the nucleus accumbens, resulting in apathy [5]. Patients with $A D$ and apathy show a blunted subjective response to dextroamphetamine challenge [7], suggesting that the use of psychostimulants increasing dopamine levels in the brain may improve 
apathy. A recent double-blind, randomized, placebo-controlled trial (DBRPCT) of methylphenidate $(n=60)$, a psychostimulant, showed that this drug improved the apathy evaluation scale-clinician version (AES-C) [8] score in patients with $A D$ as compared with a placebo [9]. However, another DBRPCT of methylphenidate $(n=60)$, which used the apathy evaluation scale-informant (AES-I) [10], did not show this effect in patients with AD [11]. Thus, the efficacy of methylphenidate remains inconclusive ( $>$ Table 1 ). The low statistical power (insufficient sample size) of these studies might make them difficult to accurately estimate the efficacy of methylphenidate. A meta-analysis can increase the statistical power of group comparisons and overcome the limitations of sample size in underpowered studies [12]. A recent meta-analysis reported that methylphenidate was superior to placebo in the improvement of apathy scales score [4]. When different studies use different scales, the Cochrane handbook recommends using random-effects models and standardized mean difference (SMD) analysis [12]. Our metaanalysis aimed to fill the gap in the literature in terms of the efficacy and safety of psychostimulants for the treatment of patients with AD. Therefore, we conducted a comprehensive systematic review and random-effect model meta-analysis (using SMD for continuous outcomes and risk ratios (RRs) for dichotomous outcomes as the response measures). This study aimed to produce conclusive evidence for the efficacy (improvement of apathy, cognitive impairment, activity of daily living, and burden of caregiving) and safety (discontinuation rate and incidence of individual adverse events) of a pooled psychostimulant group in patients with AD. We conducted this systematic review and meta-analysis by combining 2 psychostimulants (methylphenidate and modafinil) to overcome the limitations of sample size in underpowered studies.

\section{Methods}

This meta-analysis was performed according to the preferred reporting items for systematic reviews and meta-analyses guidelines [13]. The DBRPCTs using psychostimulants for AD were selected. Double-blind, randomized, placebo-controlled, crossover trials (DBRPCCOT) were included to increase the sample size for the meta-analysis. A systematic literature search was conducted according to the following aspects: patient (AD), intervention (psychostimulants), comparator (placebo), and outcomes (efficacy and safety outcomes). The review has been registered with PROSPERO (http://www.crd.york.ac.uk/PROSPERO/. CRD42018085983).

\section{Search strategy}

To identify relevant studies, 2 authors (T.K. and K.S.) independently searched databases, such as MEDLINE, Cochrane library, and Scopus, without language restrictions from the date of inception of these databases to January 6, 2019 using the following keywords: (random * ) AND (stimulant OR psychostimulant OR methylphenidate OR cathinone OR methcathinone OR cocaine OR dexmethylphenidate OR amphetamine OR methamphetamine OR 3,4-methylenedioxymethamphetamine OR dextroamphetamine OR lisdexamfetamine OR atomoxetine OR modafinil OR armodafinil OR dexamphetamine OR bupropion OR mazindol OR selegiline) AND (Alzheimer ${ }^{*}$ ). The authors also searched the following to ensure the comprehensive inclusion of randomized controlled trials and to minimize the possibility of publication bias: clinicaltrials.gov (http://clinicaltrials.gov/), ISRCTN registry (https://www.isrctn. com/), and international clinical trials registry platform (http:// www.who.int/ictrp/en/). The authors independently evaluated the inclusion/exclusion criteria and selected the relevant studies. The references of the included articles and reviews were also searched for the citations of additional relevant published and unpublished studies, including conference abstracts.

\section{Data sources, studies sections, and data extraction}

The outcomes of our study were apathy (primary) scale score (i. e., frontal systems behavior scale apathy [14] from 1 study [15], AES-I from 2 studies [11, 16], and AES-C from 1 study [9]), mini-mental state examination (MMSE) score [17], instrumental activities of daily living scale (IADL) score [18], Zarit burden interview [19] score, all-cause discontinuation rate, discontinuation due to adverse events, and incidence of individual adverse event. For evaluating apathy, 1 study [15] used frontal systems behavior scale apathy, 2 studies $[11,16]$ used both AES-I and neuropsychiatric inventory (NPI) apathy score [20], and another study [9] used AES-C. There were 2 studies using the NPI apathy score. However, because the results of these 2 studies were consistent ( Table 1 ), we did not perform a meta-analysis using only the data of NPI apathy score. Where possible, an intention-to-treat or a full analysis set population was used. Although period 1 data was not available (before crossover) for crossover studies, we used those data for meta-analysis. If the data required for meta-analysis were missing, the investigators or the industries of the relevant study were contacted and asked to provide the unpublished data. Moreover, we extracted data from previous systematic reviews and meta-analyses [4].

\section{Data analysis}

The meta-analysis was conducted using the review manager software (version 5.3 for Windows; http://tech.cochrane.org/revman), and a random-effects model was selected because of the potential heterogeneity across several studies. Continuous outcomes were analyzed using SMD with $95 \% \mathrm{Cl}$. Lower MMSE and IADL scale scores indicated a higher level of impairment or more severe symptoms; hence, the algebraic sign of the numerical scores was reversed for these scales. Dichotomous outcomes were presented as RRs. The methodological quality of the selected trials was assessed according to the risk of bias criteria in the Cochrane handbook for systematic reviews of interventions [12]. Study heterogeneity was evaluated using the heterogeneity statistic $\left(\mathrm{I}^{2}\right)$, considering $\mathrm{I}^{2} \geq$ $50 \%$ to reflect considerable heterogeneity [21]. A sensitivity analysis was conducted for primary outcomes with considerable heterogeneity, methylphenidate studies vs. modafinil study, [1] and DBRPCTs vs. DBRPCCOT [2]. Because a funnel plot is generally used only if 10 or more studies are included in the meta-analysis, we did not utilize this plot for exploring potential publication bias [12].

\section{Results}

Of the 617 studies initially identified by searching the literature, 407 were excluded after reviewing the titles and abstracts. Reviewing the full text resulted in the exclusion of 1 systematic review and 


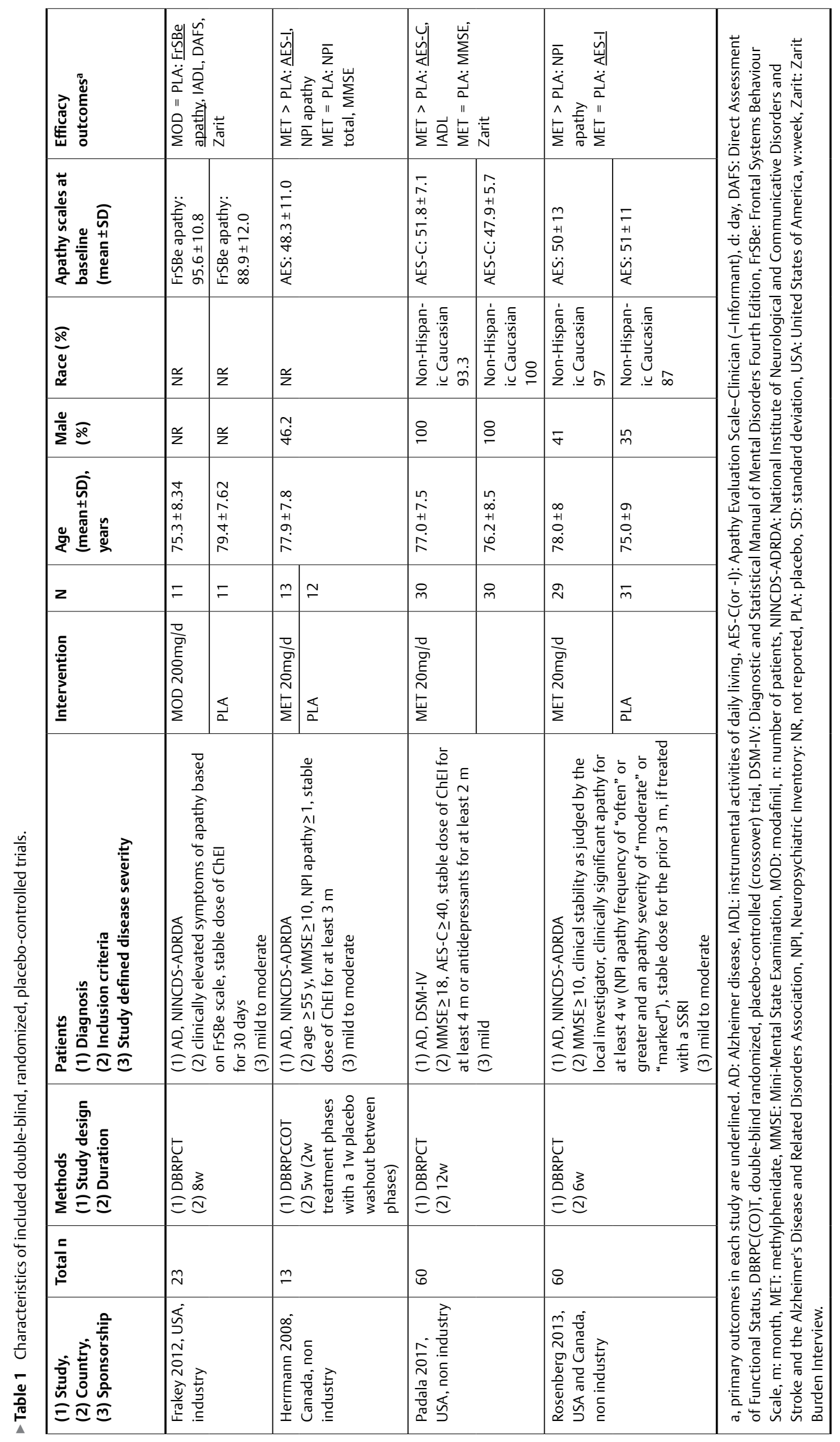


meta-analysis study [4] (Supplementary > Fig. 1S). No additional studies were retrieved further from the clinical trial registries.

\section{Risk of bias}

Three of 4 studies were DBRPCTs, and one was DBRPCCOT [16] (Supplementary - Fig. 2S). Although this DBRPCCOT did not report the data obtained before crossover, we used crossover data for the meta-analysis. The primary outcome of all the studies was improvement in the apathy scale score. One of 4 studies was sponsored by the industry [15]. Two of 4 studies did not have any high risk of bias $[9,11]$.

\section{Study selection and characteristics}

We identified 4 randomized trials that compared psychostimulants and placebo in 156 patients with AD ( $\triangleright$ Table 1). All the studies were published in English and were conducted in the USA and/or Canada. The study duration ranged 2-12 weeks. Sample sizes ranged from 13-60. All the studies used standardized diagnostic criteria, including the National Institute of Neurological and Communicative Disorders and Stroke and the Alzheimer's Disease and Related Disorders Association (3 studies) or the Diagnostic and Statistical Manual of Mental Disorders Fourth Edition (1 study). The mean age of the patients was 76.8 years.

\section{Results of the efficacy outcomes in individual studies included in the current systematic review and meta-analysis}

A study by Frakey (2012) has reported that modafinil was not superior to placebo in the improvement of the frontal systems behavior scale apathy score, IADL score, and Zarit burden interview score [15].
A study by Herrmann (2008) has shown that although methylphenidate was superior to placebo in the improvement of the AES-I and NPI apathy scores, no significant differences were observed in the MMSE score between the groups [16].

Padala (2017) has reported that although methylphenidate was superior to placebo in the improvement of the AES-C and IADL scores, no significant differences were observed in the MMSE and Zarit burden interview score between the groups [9].

In 2013, Rosenberg has shown that methylphenidate was superior to placebo in the improvement of the NPI apathy score, but no significant differences were observed in the AES-I score between the groups [11].

\section{Synthesized findings}

Combined psychostimulants were superior to placebos in the improvement of the apathy scale score $(\mathrm{SMD}=-0.63(-1.22,-0.04)$, $p=0.04, I^{2}=68 \%$, based on all included studies; ( $>$ Fig. 1 -1) and MMSE score $\left(S M D=-0.58(-1.14,-0.02), \mathrm{I}^{2}=61 \%, \mathrm{p}=0.04\right.$, based on the 3 methylphenidate studies; ( Fig. 1-2). However, no significant differences were observed with respect to other outcomes between the treatment groups (Supplementary $>$ Figs. 3S-8S).

Because we detected a considerable heterogeneity for the primary outcome, we conducted 2 sensitivity analyses. When the data of 1 modafinil study were excluded from the meta-analysis for the improvement of the apathy scales score, methylphenidate was found to be superior to placebo $(S M D=-0.82(-1.43,-0.20)$, $\left.p=0.009, I^{2}=66 \%\right)$. When the data of 1 DBRPCCOT were excluded from the meta-analysis for the improvement of the apathy scale score, combined psychostimulants were not superior to placebo $\left(\mathrm{SMD}=-0.63(-1.41,0.16), \mathrm{p}=0.12, \mathrm{I}^{2}=79 \%\right)$.

$1-1$. Forest plot of the apathy scale score

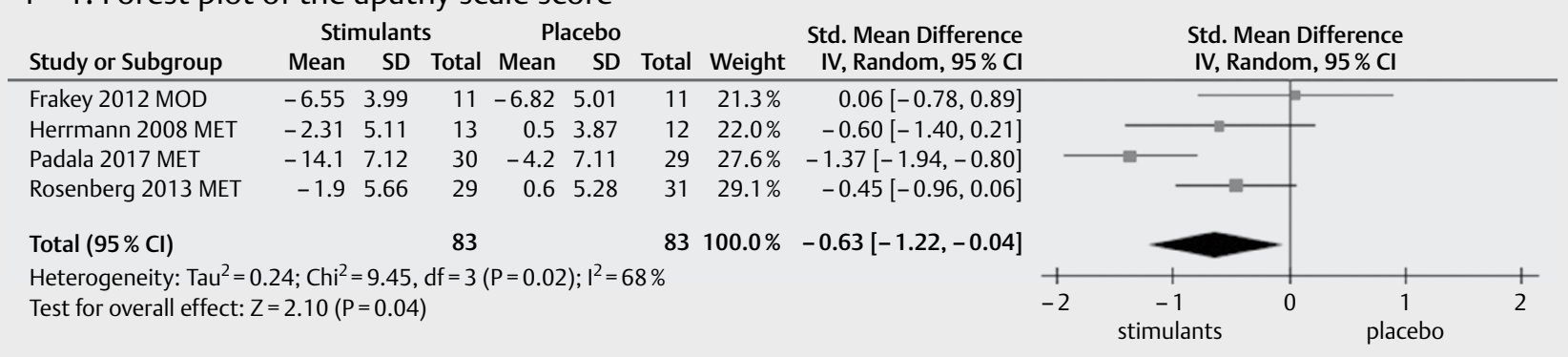

1 -2. Forest plot of the Mini-Mental State Examination score

\begin{tabular}{|c|c|c|c|c|c|c|c|c|c|}
\hline Study or Subgroup & \multicolumn{3}{|c|}{ Stimulants } & \multicolumn{2}{|c|}{ Placebo } & Total & Weight & $\begin{array}{l}\text { Std. Mean Difference } \\
\text { IV, Random, } 95 \% \text { CI }\end{array}$ & $\begin{array}{l}\text { Std. Mean Difference } \\
\text { IV, Random, } 95 \% \mathrm{Cl}\end{array}$ \\
\hline Herrmann 2008 MET & 0.58 & 2.53 & 13 & 1.08 & 2.81 & 12 & $26.4 \%$ & $-0.18[-0.97,0.61]$ & -1 \\
\hline Padala 2017 MET & -2.2 & 2.79 & 30 & 0.4 & 1.73 & 29 & $35.9 \%$ & $-1.10[-1.65,-0.55]$ & \\
\hline Rosenberg 2013 MET & -1.2 & 4.15 & 29 & 0.3 & 4.15 & 31 & $37.7 \%$ & $-0.36[-0.87,0.15]$ & \\
\hline Total $(95 \% \mathrm{Cl})$ & & & 72 & & & 72 & $100.0 \%$ & $-0.58[-1.14,-0.02]$ & \\
\hline \multicolumn{9}{|c|}{$\begin{array}{l}\text { Heterogeneity: } \text { Tau }^{2}=0.15 ; \mathrm{Chi}^{2}=5.14, \mathrm{df}=2(P=0.08) ; \mathrm{I}^{2}=61 \% \\
\text { Test for overall effect: } Z=2.02(P=0.04)\end{array}$} & 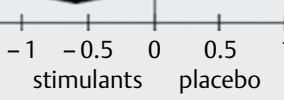 \\
\hline
\end{tabular}

Fig. 11 Forest plot of the apathy scale score. 2 Forest plot of the Mini-Mental State Examination score. (period) $95 \%$ Cls: $95 \%$ confidence intervals, IV: inverse variance, M-H: Mantel-Haenszel, MET: methylphenidate, MOD: modafinil, SD: standard deviation, Std mean difference: standardized mean difference 


\section{Discussion}

We performed a comprehensive systematic review and meta-analysis to obtain robust evidence of the efficacy and safety of the combined psychostimulants in patients with AD. Methylphenidate was found to improve apathy and cognitive impairment in patients with AD. It is a drug approved by the U.S. Food and Drug Administration [22] for the treatment of attention deficit hyperactivity disorder and narcolepsy. Methylphenidate blocks the reuptake of norepinephrine and dopamine into the presynaptic neuron and increases the release of these monoamines into the extraneuronal space [22]. The pathophysiology of apathy and cognitive impairment includes hypofunction of dopamine neurons in the brain [5,6]. Methylphenidate can improve these symptoms by restoring the normal function of dopamine neurons. Although the study duration included in the current meta-analysis was short, no significant differences were observed in discontinuation rate as well as incidence of at least 1 adverse event between psychostimulants and placebo. However, the meta-analysis did not include other safety outcomes, as insufficient data was available on these outcomes.

Because we detected considerable heterogeneity in the primary outcome, we conducted 2 sensitivity analyses; however, these did not reveal any confounding factors. The considerable heterogeneity might have been observed because of small sample sizes. Because we did not utilize funnel plot for exploring potential publication bias, our study results might include a publication bias. Moreover, because the number of RCTs and patients included in our meta-analysis was small, we cannot rule out a "small study effect," in which smaller studies tend to show larger treatment effects than larger studies [23].

\section{Limitations}

First, the number of studies and patients included in this metaanalysis are limited. Second, because all the included studies had short trial durations, we could not determine whether psychostimulants would have long-term effects on apathy and cognitive impairment. Although the use of psychostimulants poses a risk of drug dependence, cardiovascular disease, psychiatric symptoms, such as psychosis and mania, as well as seizures [22], the current systematic review and meta-analysis did not evaluate the association between psychostimulants and these risks in patients with AD. Moreover, we did not perform a meta-analysis in terms of safety outcomes other than discontinuation rate and incidence of at least 1 adverse event. Third, because a funnel plot is generally used only if 10 or more studies are included in the meta-analysis, we did not utilize such method for exploring potential publication bias [12].

\section{Conclusions}

Our results suggest that methylphenidate is effective in treating apathy and cognitive impairment in patients with AD. However, the number of patients and studies included in the current systematic review and meta-analysis was limited. Moreover, the duration of the included studies was short. Therefore, we considered that a long-term study with larger sample size must be conducted to obtain robust results.

\section{Contributors}

All authors had full access to all study data and are responsible for the integrity of the data and the accuracy of any data analysis. All authors drafted the final manuscript.

\section{Acknowledgments}

None.

\section{Funding}

This study was supported by an unrestricted grant from Daiichi Sankyo. Daiichi Sankyo had no role in study design, data collection and analysis, decision to publish, or preparation of the manuscript. All authors declare no conflicts of interest.

\section{Competing Interests}

The authors have declared that there are no conflicts of interest in relation to the subject of this study. We have had the following interests within the past 3 years. Dr. Kishi has received speaker's honoraria from Daiichi Sankyo, Dainippon Sumitomo, Eisai, Janssen, Kyowa, Meiji, MSD, Otsuka, Tanabe-Mitsubishi and Yoshitomi, and has received a Health Labor Sciences Research Grant, Grant-in-Aid for Scientific Research (C), and a Fujita Health University School of Medicine research grant. Dr. Sakuma has received speaker's honoraria from Otsuka and Torii and has received a Grant-in-Aid for Young Scientists. Dr. Iwata has received speaker's honoraria from Astellas, Dainippon Sumitomo, Eli Lilly, GlaxoSmithKline, Janssen, Yoshitomi, Otsuka, Meiji, Shionogi, Novartis, and Pfizer and has had research grants from Daiichi Sankyo, Dainippon Sumitomo, Meiji, and Otsuka.

\section{References}

[1] Rajan KB, Weuve J, Barnes LL et al. Prevalence and incidence of clinically diagnosed Alzheimer's disease dementia from 1994 to 2012 in a population study. Alzheimers Dement 2019; 15: 1-7

[2] Scheltens P, Blennow K, Breteler MM et al. Alzheimer's disease. Lancet 2016; 388: 505-517

[3] Kishi T, Matsunaga S, Iwata N. The effects of memantine on behavioral disturbances in patients with Alzheimer's disease: A meta-analysis. Neuropsychiatr Dis Treat 2017; 13: 1909-1928

[4] Ruthirakuhan MT, Herrmann N, Abraham EH et al. Pharmacological interventions for apathy in Alzheimer's disease. Cochrane Database Syst Rev 2018; 5: CD012197

[5] Martorana A, Koch G. Is dopamine involved in Alzheimer's disease? Front Aging Neurosci 2014; 6: 252

[6] Nobili A, Latagliata EC, Viscomi MT et al. Dopamine neuronal loss contributes to memory and reward dysfunction in a model of Alzheimer's disease. Nat Commun 2017; 8: 14727

[7] Lanctot KL, Herrmann N, Black SE et al. Apathy associated with Alzheimer disease: Use of dextroamphetamine challenge. Am J Geriatr Psychiatry 2008; 16: 551-557

[8] Clarke DE, Reekum R, Simard M et al. Apathy in dementia: An examination of the psychometric properties of the apathy evaluation scale. J Neuropsychiatry Clin Neurosci 2007; 19: 57-64 
[9] Padala PR, Padala KP, Lensing SY et al. Methylphenidate for apathy in community-dwelling older veterans with mild Alzheimer's disease: A double-blind, randomized, placebo-controlled trial. Am J Psychiatry 2018; 175: 159-168

[10] Marin RS, Biedrzycki RC, Firinciogullari S. Reliability and validity of the apathy evaluation scale. Psychiatry Res 1991; 38: 143-162

[11] Rosenberg PB, Lanctot KL, Drye LT et al. Safety and efficacy of methylphenidate for apathy in Alzheimer's disease: a randomized, placebo-controlled trial. J Clin Psychiatry 2013; 74: 810-816

[12] Higgins J, Green S. Cochrane Handbook for Systematic Reviews of Interventions Version 5.1.0 [updated March 2011]. In: Collaboration TC ed. http://handbookcochraneorg2011

[13] Moher D, Liberati A, Tetzlaff J et al. Preferred reporting items for systematic reviews and meta-analyses: the PRISMA statement. BM] 2009; 339: b2535

[14] Grace J, Malloy P. Frontal Systems Behavior Scale (FrSBe): Professional Manual. Psychological Assessment Resources; Lutz, Florida: 2001

[15] Frakey LL, Salloway S, Buelow M et al. A randomized, double-blind, placebo-controlled trial of modafinil for the treatment of apathy in individuals with mild-to-moderate Alzheimer's disease. J Clin Psychiatry 2012; 73: 796-801
[16] Herrmann N, Rothenburg LS, Black SE et al. Methylphenidate for the treatment of apathy in Alzheimer disease: prediction of response using dextroamphetamine challenge. J Clin Psychopharmacol 2008; 28: 296-301

[17] Folstein MF, Robins LN, Helzer JE. The mini-mental state examination. Arch Gen Psychiatry 1983; 40: 812

[18] Lawton MP, Brody EM. Assessment of older people: Self-maintaining and instrumental activities of daily living. Gerontologist 1969; 9: 179-186

[19] Zarit SH, Reever KE, Bach-Peterson J. Relatives of the impaired elderly: Correlates of feelings of burden. Gerontologist 1980; 20: 649-655

[20] Cummings JL, Mega M, Gray K et al. The neuropsychiatric inventory: Comprehensive assessment of psychopathology in dementia. Neurology 1994; 44: 2308-2314

[21] Higgins JP, Thompson SG, Deeks J] et al. Measuring inconsistency in meta-analyses. BMJ 2003; 327: 557-560

[22] FDA Methylphenidate. https://wwwaccessdatafdagov/drugsatfda_ docs/label/2007/010187s069,018029s040,021284s011lblpdf

[23] Moreno SG, Sutton A], Ades AE et al. Assessment of regression-based methods to adjust for publication bias through a comprehensive simulation study. BMC Med Res Methodol 2009; 9: 2 